\title{
Object detection framework for multiclass food object localization and classification
}

\begin{abstract}
Detecting the instances of an object-class is a very important and crucial task in computer vision system prior to obtaining any further information. To determine the location of the object instances possess several challenges resulted from the object and image variations. In this paper, we propose a recognition framework for multiclass object detection to localize and classify the food objects to address the problem of searching multiclass objects. A typical food object, to compare to the other objects has non-rigid deformation and suffers from very large intraclass variance and too little inter-class similarities. To strive a better recognition performance while designing this framework, the optimal food recognition components comprising localization, feature extraction and classification strategy were discovered through a literature review. Besides that, the problems that are still remaining in this area critically discussed along with research direction that should be put into concentration for the future research.
\end{abstract}

Keyword: Food object detection; Image classification; Multiclass object detection; Object detection. 